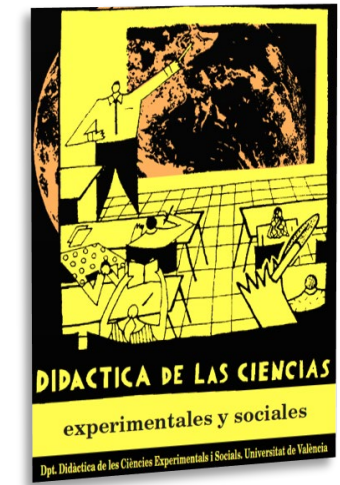

\title{
Situación de la enseñanza de las ciencias por indagación en los planes de estudio de Grado de Maestro de Educación Primaria en España
}

\author{
Status of inquiry-based science education in \\ the Spanish curricula of the Degrees in \\ Primary School Education
}

DOI: $10.7203 / D C E S .39 .17855$

\author{
Sandra Pilar Tierno \\ Universidad Católica de Valencia, sp.tierno@ucv.es \\ ORCID iD: https://orcid.org/0000-0003-3191-297X \\ Paula Tuzón \\ Universitat de València, paula.tuzon@uv.es \\ ORCID iD: https://orcid.org/0000-0003-1344-3751 \\ Jordi Solbes \\ Universitat de València, jordi.solbes@uv.es \\ ORCID iD: https://orcid.org/0000-0002-8220-209X \\ Valentín Gavidia \\ Universitat de València, valentin.gavidia@uv.es \\ ORCID iD: https://orcid.org/0000-0001-9153-147X
}

\begin{abstract}
RESUMEN: En el presente trabajo se analizan 84 guías docentes de asignaturas de ciencias experimentales y/o su didáctica impartidas en el Grado en Maestro en Educación Primaria de universidades españolas. El análisis de la información contenida en ellas nos permite conocer la formación que proponen las guías docentes de asignaturas científicas de dicho grado y hasta qué punto la enseñanza de la ciencia por indagación está presente en su plan de formación. Los resultados indican una escasa formación científica de los futuros maestros y con poca presencia de la indagación.
\end{abstract}

Palabras ClaVe: enseñanza de las ciencias, maestros en formación, indagación, Educación Primaria, guía docente.

ABSTRACT: The present study analyses 84 teaching guides of subjects about experimental sciences and/or their teaching of the Degree in Primary School Education from Spanish universities. This analysis addresses the science education proposed in the teaching guides of these subjects for pre-service Primary School teachers and the presence of inquiry-based science education in their curricula. The results show little science training for pre-service teachers and a low presence of inquiry-based science education in their curricula.

KEYWORDS: science education, pre-service teachers, inquiry, Primary Education, teaching guides.

Fecha de recepción: julio de 2020

Fecha de aceptación: octubre de 2020

Este trabajo forma parte del proyecto "Estudio sobre la enseñanza de las ciencias en educación infantil y primaria. Propuestas de mejora” PID2019-105320RB-I00, financiado por el Ministerio de Ciencia e Innovación. 


\section{INTRODUCCIÓN}

La Didáctica de las Ciencias tiene por objeto tratar los problemas referidos a la enseñanzaaprendizaje de las ciencias en todos sus niveles y ámbitos, uno de los cuales es la formación del profesorado. La cuestión sobre qué debe saber y qué debe saber hacer un docente de ciencias se aborda desde hace varias décadas (Shulman, 1986; Gil, 1991; Furió y Carnicer, 2002; García Barros, 2016). Así, por un lado, un profesor de ciencias debe conocer tanto la materia en cuestión como las relaciones entre la ciencia, la tecnología y la sociedad, la historia de la ciencia o algunos desarrollos científicos actuales. Por otro lado, es imprescindible que conozca qué ciencia enseñar y para qué, cómo enseñar ciencia, cómo los alumnos la aprenden, cómo preparar y/o dirigir las actividades del aula o cómo evaluar. Esto nos daría, pues, un profesor de ciencias preparado desde el punto de vista de la materia científica y de las cuestiones didácticas relacionadas.

Sin embargo, la integración de estos dos aspectos no siempre ocurre. Es una realidad el nivel científico básico que poseen los alumnos del Grado en Maestro (Cañal, 2000; Montero-Pau, Tierno y Tuzón, 2017; Verdugo-Perona, Solaz-Portolés y Sanjosé, 2016, 2019), y esto lleva, a menudo, a que las facultades de magisterio o educación incluyan asignaturas de ciencias experimentales que complementen el nivel científico de los alumnos. Ahora bien, si la formación de la materia científica fuera exclusiva, esto podría llevarles a no reflexionar sobre las herramientas didácticas que necesitarán para impartir los contenidos científicos que van aprendiendo (Esteve, 2006). En consecuencia, esto suscita preguntas al respecto que deben ser respondidas. ¿Cómo se imparte la formación científica a los maestros? ¿Está integrada la parte didáctica con la parte científica? ¿En qué cursos reciben los alumnos del Grado de Maestro las asignaturas científicas? ¿La formación que se imparte a los futuros maestros es mayoritariamente de contenidos científicos o didácticos? ¿Cómo es la visión general de estas asignaturas de ciencias, disciplinar o multidisciplinar?

Por otro lado, la recomendación de una enseñanza de las ciencias basada en la indagación (ECBI) está más que aceptada, sin embargo, consideramos que la indagación no debe abordarse únicamente con el foco puesto en la educación de niveles pre-universitarios, sino que la indagación debe también estar presente en la formación científica y didáctica de los futuros maestros. Es más, en la ECBI, el rol del profesor que la implementa es importante por lo que debe asegurarse una integrada y completa formación científica y didáctica del profesorado (Cañal, 2012). Sin embargo, algunos estudios destacan la falta de preparación entre el profesorado para llevar a la práctica esta metodología en sus aulas debido al poco conocimiento científico del que disponen (Murphy, Neil y Beggs, 2007; Forbes y Davis, 2010), o sus dudas acerca de la metodología científica (Vílchez González y Bravo Torija, 2015).

Asimismo, estudios en los que se llevan a cabo entrevistas a maestros en activo (MartínezChico, López-Gay, Jiménez Liso y Acher, 2013; Montero-Pau y Tuzón, 2017) y a formadores de maestros (Martínez-Chico, Jiménez-Liso y López-Gay, 2014a), revelan la petición de una formación inicial en la que se traten los conceptos científicos importantes que después tendrán que desarrollar y que experimenten las metodologías de enseñanza que más tarde podrán llevar a la práctica en sus aulas. Los maestros en activo muestran, en general, una falta de comprensión acerca de lo que significa enseñar a los alumnos a investigar (Capps y Crawford, 2013; Montero-Pau y Tuzón, 2017), al igual que los maestros en formación inicial presentan dudas acerca de cómo enseñar ciencia mediante indagación (Toma, Greca y Meneses-Villagrá, 2017).

No obstante, existen ya en la literatura propuestas de aplicación de esta metodología en la formación inicial de maestros (Martínez-Chico, López-Gay y Jiménez-Liso, 2014b; Vílchez González y Bravo Torija, 2015; Garrido Espeja y Couso, 2017). También hay estudios recientes sobre la formación que se propone en las guías didácticas (García Barros, 2016), que pretendemos actualizar. Sin embargo, no hay estudios sobre si la metodología por indagación está declarada en las guías docentes de las asignaturas de ciencias experimentales y su didáctica del plan de formación 
inicial de los maestros y, por ello, nos preguntamos en qué grado la indagación aparece reflejada explícitamente en sus guías docentes.

Esta cuestión, junto a las anteriores referentes a la formación de los maestros, son a las que pretendemos dar respuesta en el presente trabajo y, para ello, establecemos los siguientes objetivos: 1) Realizar un análisis de la formación en Ciencias Experimentales y/o su Didáctica que proponen las guías docentes de las asignaturas relacionadas y 2) Determinar el grado de presencia de la indagación en estas guías docentes del Grado de Maestro en Educación Primaria de las universidades públicas españolas.

Con el desarrollo del presente estudio esperamos, primero, actualizar la visión general de la formación científica y de didáctica de las ciencias que presentan las guías docentes de las universidades españolas, para entender el modelo docente que se propone actualmente en las facultades de educación y/o magisterio. En segundo lugar, aportamos una aproximación a la presencia de la indagación en las guías docentes de los planes de estudios de los futuros maestros, la cual puede suponer un indicio de los cambios necesarios en la enseñanza-aprendizaje de las ciencias mediante indagación para estos futuros docentes.

\section{MARCO TEÓRICO}

\subsection{La formación del profesorado de ciencias}

La formación de maestros de ciencias se nutre de la respuesta a dos cuestiones: qué deben saber y qué deben saber hacer los docentes de ciencias. Estas preguntas no pueden resolverse únicamente mediante la suma aditiva de la materia científica y los aspectos didácticos de la misma, sino que es resultado de un ensamblaje más complejo. Por ello, esta cuestión lleva abordándose desde varias décadas atrás, partiendo del término de Conocimiento didáctico del contenido, introducido por Shulman (1986).

Como consideran Gil (1991) y Furió y Carnicer (2002), el profesorado de ciencias no debe solamente conocer los contenidos disciplinares y su didáctica, sino que, además, es necesario que pueda y sepa realizar una autocrítica de su enseñanza, se implique en actividades de innovación y/o investigación, y que cuestione su pensamiento docente espontáneo. Esto nos lleva a la elaboración de un concepto más complejo e integral como es el Conocimiento Profesional del Profesor (CPP), que contempla diferentes aspectos como señalan Solbes, Domínguez-Sales, Fernández-Sánchez, Furió, Cantó y Guisasola (2013); Solbes, Fernández-Sánchez, Domínguez-Sales y Cantó (2018):

a. el conocimiento de la disciplina, incluyendo la naturaleza de la ciencia, la historia de la ciencia, las interacciones ciencia, tecnología y sociedad y la selección y secuenciación de los contenidos didácticos adecuados;

b. el conocimiento pedagógico que atiende a aspectos más generalistas como la gestión del aula, los objetivos de la educación, la introducción y utilización de las TICs, etc; y

c. el conocimiento didáctico del contenido, que atiende, a su vez, a los cinco aspectos citados por Magnusson et al (1999), que incluyen los objetivos y finalidad de la enseñanza de las ciencias, los programas curriculares específicos y los objetivos según la normativa y legislación vigente, los estudiantes y sus características, la evaluación y las estrategias instruccionales.

Sin embargo, para que los futuros maestros puedan desarrollar su propio conocimiento didáctico del contenido, en la formación inicial que reciban deberían integrarse el conocimiento académico, el práctico y las concepciones personales que tenga el futuro profesorado (Mellado, 2003), porque cada futuro maestro tiene un conocimiento profesional previo a la formación inicial, que determinará también su manera de actuar en el aula (Rivero, Martín del Pozo, Solís y Porlán, 2017). Es lo que Porlán y Rivero (1998) denominan conocimiento tácito profesional, y es resultado de la experiencia docente previa con la que los futuros maestros acuden a las aulas universitarias, derivada de su tiempo como alumnos. Este conocimiento profesional previo deberá evolucionar hasta el conocimiento 
profesional deseable propuesto por sus formadores, y que se entiende debe ser coherente con los resultados de la investigación e innovación (Porlán, Rivero y Martín del Pozo, 1998; Porlán, 2018).

Por otro lado, debe tenerse en cuenta que hay multitud de competencias que debe poseer un profesor de ciencias para desempeñar correctamente su tarea docente, tales como tomar decisiones acerca de qué enseñar y cómo, saber desarrollar la acción educativa favoreciendo el aprendizaje e interés de los alumnos, evaluar la enseñanza y promover su propio desarrollo profesional (García Barros, 2016). Solamente una formación que conjugue todo lo anterior podrá formar adecuadamente al profesorado de ciencias.

Ahora bien, la manera en la que se plantee la integración anterior en el plan de formación de los maestros puede realizarse según diferentes modelos. Un modelo consecutivo, como el que se sigue en España para formar a los profesores de secundaria, propone primero una formación disciplinar y posteriormente, la didáctica. En un modelo simultáneo, estos dos aspectos se estudian a la vez. Tal y como refería Esteve (2006), Europa estaba optando por este modelo para la formación inicial del profesorado, permitiendo que se forme a la vez en los contenidos disciplinares y en los didácticos. El modelo simultáneo, comenta el autor, evitaría que los futuros docentes se formen una falsa identidad de especialista en ciencias, así como les permitiría reflexionar sobre qué herramientas necesitarán en un futuro para impartir clases de ciencias, ayudándoles en la formación de una identidad como docentes (Esteve, 2006). En consecuencia, conocer cómo se integran las asignaturas científicas y/o de su didáctica en el plan de estudios del futuro profesorado puede permitirnos inferir el modelo de formación de los maestros de ciencias preferido por los centros universitarios españoles.

\subsection{La enseñanza de las ciencias basada en la indagación}

En la enseñanza de las ciencias es clave incorporar el aprendizaje de los procedimientos científicos al de los propios contenidos conceptuales y actitudinales (Akerson y Hanuscin, 2007; Harlen, 2009; Minner, Levy, y Century, 2010; Furtak, Seidel, Iverson y Briggs, 2012; Louca y Zacharia, 2012; Murphy, Smith, Varley y Razi, 2014; BOE, 2014). El aprendizaje de los métodos en ciencias, esto es, de los momentos y destrezas involucradas en una investigación, forman parte de lo que se denomina enseñanza de las ciencias basada en la indagación (ECBI).

La indagación cobra especial relevancia en educación primaria. Por una parte, atiende procedimientos muy relevantes para esta etapa (Harlen, 2009): observación, clasificación, agrupación, manipulación directa, toma de medidas, montaje, etc. Por otra parte, aspectos como la elaboración de hipótesis o las primeras impresiones acerca del fenómeno, permiten a los niños expresar sus ideas previas (Keynes, Driver, Guesne y Tiberghien, 1985; Garbett, 2003; Allen, 2010; Vosniadou, 2013), las cuales son muy importantes en la enseñanza de las ciencias; por tanto, implementar una metodología que permita a los niños expresarlas y confrontarlas es muy ventajoso para su aprendizaje.

Sin embargo, la definición de la ECBI no es sencilla, dado que dentro de esta denominación se enmarcan tantas intervenciones didácticas que es difícil encontrar dentro de la literatura publicada una definición aceptada por todos (Hayes, 2002; Capps y Crawford, 2013; Couso, 2014). Esta indefinición lleva, por un lado, asociada la existencia de varios tipos de indagación, desde una indagación abierta en la que el alumno decide la pregunta de investigación y el camino a seguir en la búsqueda de su respuesta, hasta una indagación guiada que simplemente responda preguntas propuestas en clase (Windschitl, 2003; Kirschner, Sweller y Clark, 2006; Aguilera Morales, MartínPáez, Valdivia-Rodríguez, Ruiz-Delgado, Williams-Pinto, Vílchez-González y Perales-Palacios, 2018). Existe en la literatura una discusión al respecto, no sólo de la concepción de la indagación a la hora de implementarla en las aulas sino también de su efectividad dependiendo de cuál sea dicha implementación (Kirschner et al., 2006 y Sweller, Kirschner y Clark, 2007; Romero-Ariza, 2017). Existe también un debate interesante sobre si la indagación consiste en el aprendizaje de los métodos científicos o si consiste en aprender a través de los métodos - se entiende, habiéndolos aprendido con 
antelación o aprovechando el mismo proceso para hacerlo - (Kirschner et al. 2006; Sweller et al., 2007). Cierto es que el nivel de complejidad de esta discusión puede diferir dependiendo del nivel educativo al cual se está aplicando la indagación. En el marco de la educación primaria, entendemos la indagación en el sentido en que lo hace la autora Wynne Harlen (Harlen, 2009) y en el modo en el que enmarca sus investigaciones didácticas a lo largo de esta etapa educativa: planteando investigaciones guiadas a los niños, donde se responde una pregunta de investigación autocontenida y relevante en el currículum con objetivos de aprendizaje específicos tanto relativos a los métodos como al propio concepto.

Sin embargo, para el análisis de su presencia en los programas docentes, en este artículo intentaremos establecer a partir de la bibliografía unos indicadores de la indagación que pueden ser unos ingredientes mínimos comunes en todas sus concepciones. Así, tendremos en cuenta que, para considerar que el alumnado sabe indagar, éste debe conocer las destrezas relacionadas con las distintas etapas de una investigación en torno a una pregunta: la observación, la emisión de hipótesis, el diseño de una prueba fiable, la ejecución de un experimento, la interpretación de los datos, las conclusiones y la comunicación y reflexión (Pedaste et al., 2015). De manera que, si bien es cierto que la presencia de estos procedimientos en las guías docentes no garantiza una enseñanza por indagación podemos, desde el punto de vista opuesto, considerar que una enseñanza por indagación, al menos, incluirá estos procedimientos. Es desde esta postura desde la que se enfoca el presente análisis de la formación en indagación en la enseñanza de las ciencias a futuros maestros y maestras: si en las guías docentes no aparecen estos indicadores, difícilmente se estará impartiendo indagación. De todos modos, se detalla en el apartado siguiente la metodología seguida para la consecución de este segundo objetivo.

\section{Metodología}

Se lleva a cabo un estudio descriptivo y transversal realizado a partir de la información obtenida de las guías docentes de las asignaturas impartidas en el Grado de Maestro de Educación Primaria de diferentes universidades públicas españolas, disponibles en las páginas web de las facultades correspondientes. Las guías docentes de las asignaturas son el documento por el que se establece públicamente el acuerdo de enseñanza-aprendizaje entre el profesor y el alumnado, sirven para que éste pueda conocer todos los detalles de la asignatura a cursar y organizarse, pero también para que el profesorado pueda describir su planificación, la carga de trabajo, la metodología, el contenido, la evaluación, la bibliografía o la relación de competencias (García Martín, 2010). En consecuencia, el análisis de las guías docentes de las diferentes asignaturas de ciencias experimentales y/o su didáctica en dicho Grado en España muestra el compromiso que los diferentes departamentos universitarios y, por lo tanto, el sistema educativo superior, adquiere con nuestros futuros maestros. Analizarlas es, pues, imprescindible para conocer la propuesta de formación científica que reciben los maestros en sus estudios de grado.

La muestra la constituyen 21 universidades públicas españolas: Universidad del País Vasco/Euskal Herriko Unibertsitatea, Universidad de Alicante, Universitat Autònoma de Barcelona, Universidad Autónoma de Madrid, Universitat de Barcelona, Universidad de Castilla-La Mancha, Universidad Complutense de Madrid, Universidad de Extremadura, Universitat de Girona, Universitat de les Illes Balears, Universitat Jaume I, Universidad de La Laguna, Universidad de Málaga, Universidad de Oviedo, Universidad Rey Juan Carlos, Universidad de Salamanca, Universidad de Santiago de Compostela, Universidad de Sevilla, Universitat de València, Universidad de Valladolid y Universidad de Zaragoza, lo que se traduce en un total de 84 asignaturas (de las cuales 55 de ellas son de carácter obligatorio), de diferentes cursos, materias y número de créditos. Las asignaturas corresponden al curso académico 2016/2017. La elección de las universidades ha respondido a la intención de mostrar una representación de prácticamente todas las comunidades autónomas, aunque la técnica de muestreo utilizada no ha sido probabilística. 
1) Para responder al primer objetivo, se han analizado las guías docentes de todas las asignaturas de Ciencias Experimentales (CCEE) y/o su Didáctica impartidas en las facultades en las que se imparte en título de Grado en Educación Primaria de las universidades de la muestra. Las variables analizadas en cada guía docente han sido: nombre de la universidad y de la asignatura, número de créditos, curso en el que se imparte, carácter de la asignatura (básica, obligatoria u optativa), contenido de la asignatura (Ciencias Experimentales, Didáctica de las Ciencias Experimentales o Ciencias Experimentales y su Didáctica) y perspectiva de la materia impartida (Genérica o Específica). Además del análisis anterior, se han contabilizado aquellas asignaturas que abordan temas de interés por el contexto socioeducativo actual, como la Educación para la Salud, la Sostenibilidad, TICs y la Educación Ambiental. Estos aspectos se han englobado dentro de una categoría denominada Transversal.

En lo referente al carácter de la asignatura, clasificamos las asignaturas estudiadas en básicas, obligatorias y optativas siguiendo la clasificación que aparece en los planes de estudio del grado de Maestro en Educación Primaria. Su calificación de Básica se debe a que debe cursarse obligatoriamente por el alumnado de los primeros cursos de grado y el contenido no tiene por qué ser específico de la titulación, pudiendo aparecer en diferentes titulaciones (solo ocurre en un caso). Sin embargo, los contenidos de las asignaturas Obligatorias sí que están relacionados con las competencias específicas de la titulación en cuestión.

Por lo que respecta al contenido, clasificamos las asignaturas según si los aspectos abordados son únicamente contenidos científicos (categoría de Ciencias Experimentales, como por ejemplo las asignaturas Educación científica para maestros, Fundamento de Ciencias de la Materia o Física Básica para la Formación de Maestros), si son únicamente aspectos didácticos de las Ciencias (categoría de Didáctica de las Ciencias Experimentales, como por ejemplo las asignaturas Didáctica del medio biológico y geológico o Enseñanza y Aprendizaje de las Ciencias Experimentales I) o bien si incluyen ambos tipos de contenidos (categoría de Ciencias Experimentales y su Didáctica, cuyos ejemplos serían Fundamentos y didáctica de física o Educación Ambiental y su Didáctica). La clasificación de la materia en estas tres categorías se ha realizado atendiendo únicamente los puntos incluidos en el Temario o Contenidos (a partir de ahora, usaremos el término Temario para evitar confusiones con Contenido disciplinar), de las guías docentes. Se ha optado por analizar este apartado para hacer una simplificación. No obstante, el Temario es lo más concreto de una guía docente, es el lugar en el que se transcribe el compromiso de lo que se va a hacer en el aula y, fundamentalmente, es el único apartado de la guía que siempre aparece en las guías analizadas, independientemente del formato que utilicen.

Por último, la variable perspectiva se refiere a cómo es el enfoque de la materia impartida, si se organiza alrededor de una sola rama de la ciencia o de manera más global. Agrupamos dentro de la categoría de Perspectiva Genérica aquellas asignaturas que tratan la ciencia de una forma general o bien aspectos de varios campos de la ciencia, tales como El medio natural I. Física, Química y su Didáctica, Didáctica de las Ciencias Experimentales o Ciencias Naturales para Maestros. Incluimos en una Perspectiva Específica, las materias que sólo abordan aspectos de una sola rama de la ciencia, como física, química, biología o geología, ya sea impartiendo contenidos científicos o didácticos.

2) Para conseguir el segundo objetivo, que trata de comprobar la presencia de la indagación en las guías docentes, se ha realizado una búsqueda de la aparición de palabras clave analizadas en su propio contexto, llevando a cabo un análisis del contenido en todo el texto de la guía. Las palabras de búsqueda, que hacen referencia a la metodología científica, han sido las siguientes (entre paréntesis se especifica la raíz de búsqueda): indagación (indag*), investigación (investig*), observación (observ*), hipótesis, variable, datos, gráficas, prueba, experimento (experim*), diseño experimental (diseño experim*), problema, pregunta, comunicar (comunic*) y reflexión (reflex*). A la vista de esta consideración "de mínimos", somos conscientes de que su presencia no asegura que la ECBI sea tratada. Debe aclararse que únicamente estamos analizando si aparecen los ítems que caracterizan la metodología científica en la guía, entendidos como condición necesaria pero no suficiente, para la 
presencia de la indagación. En ningún caso se analiza cómo se hace indagación, propósito que requeriría de más instrumentos de análisis. Así pues, únicamente estamos comprobando si la indagación está presente, ya que si aparecen los términos de búsqueda en las guías significa que es posible que se dé indagación en el aula, pero si no aparecen difícilmente puede ocurrir.

El proceso de análisis de las 82 guías fue dinámico. En una primera fase, el grupo de investigación acordó los criterios de inclusión de las palabras clave y uno de sus miembros realizó el análisis de las guías, que después discutió con los demás. Las discrepancias o dudas se resolvieron mediante la discusión de los cuatro investigadores hasta el acuerdo por mayoría. En todo momento, se tuvo en cuenta el contexto o frase en la que el ítem en cuestión estaba incluido; no se realizó un análisis automatizado de la sola presencia de la palabra clave, puesto que esto no habría contemplado si el ítem se refería exactamente a la actividad científica. Además, se han omitido aquellos resultados que no hacían alusión directa a procedimientos científicos $\mathrm{y}$, por lo tanto, que no estuvieran relacionados con el tema de estudio.

Por último, el análisis del contenido se ha realizado identificando el lugar de la guía en el cual aparece la palabra o raíz y si ésta hace alusión únicamente al Contenido disciplinar o también remite a la parte Didáctica. Para simplificar los resultados encontrados, se ha considerado simplemente si los ítems aparecían en el apartado de Temario de la asignatura o si aparecían en apartados diferentes, tales como competencias, resultados de aprendizaje..., toda vez que estos apartados no existen en todas las guías.

\section{RESULTADOS Y DISCUSIÓN}

\subsection{Primer objetivo: descripción de las guías docentes de las asignaturas de ciencias y didáctica de las ciencias}

Respecto a la variable contenido de la asignatura, encontramos 27 asignaturas $(32,1 \%)$ de Didáctica de las CC. Experimentales, $41(48,8 \%)$ de CC. Experimentales y su didáctica, en las que se imparten ambos tipos de materias en la misma asignatura, y 16 asignaturas $(19,0 \%)$ con enfoque únicamente disciplinar. Valores cercanos encontramos en el trabajo realizado por García Barros (2016), en el que los porcentajes encontrados en sus 86 asignaturas analizadas difieren en cuanto a que el porcentaje de asignaturas de únicamente contenido didáctico es menor que el nuestro y el de únicamente contenido disciplinar es mayor. Entendemos las discrepancias por diferencia en las muestras elegidas.

Esta distribución de asignaturas de predominio didáctico y generalista es esperable, dado que se está analizando el plan de estudios del Grado de Maestro. Además, de este resultado puede intuirse la preferencia por ofrecer asignaturas que conjuguen los contenidos didácticos con los contenidos científicos, ya que casi la mitad de las asignaturas se organizan de dicha manera, siguiendo un modelo simultáneo de formación como el defendido por Esteve (2006). La distribución por curso de las asignaturas se puede apreciar en el Gráfico 1, donde se observa que la mayoría de las asignaturas donde se imparte Ciencias y su Didáctica suelen ofrecerse en los cursos de $2^{\circ}$ y $3^{\circ}$, mientras que las asignaturas exclusivas de didáctica de las ciencias se imparten predominantemente en los cursos de $3^{\circ}$ y $4^{\circ}$. Los contenidos disciplinares se encuentran más distribuidos en los diferentes cursos, aunque es en $1^{\circ}$ cuando son más frecuentes. El curso Sin definir que aparece en el gráfico se corresponde con asignaturas optativas que no tienen un curso definido asignado. Resultados esperables, dado que los primeros cursos del Grado en Maestro en Educación Primaria suelen impartirse asignaturas de didáctica general o psicología, y las especialidades se abordan en cursos superiores. Esto es ventajoso desde el punto de vista del alumno del grado puesto que le permite tener una base en didáctica general sobre la que trabajar los aspectos de didáctica específica y entender mejor sus particularidades. Por su parte, la distribución de las asignaturas de únicamente CCEE se puede entender desde el hecho de que algunas de estas asignaturas se proponen en los primeros cursos para ofrecer una nivelación de 
ciencias a los alumnos que acceden al grado, pero también hay otras asignaturas que se ofertan de forma optativa para una mayor profundización en aspectos científicos particulares. Éstas últimas asignaturas no tienen ya, por qué ofertarse en cursos inferiores.

GRÁFICO 1. Distribución (frecuencia absoluta) de las asignaturas según contenido y curso de impartición

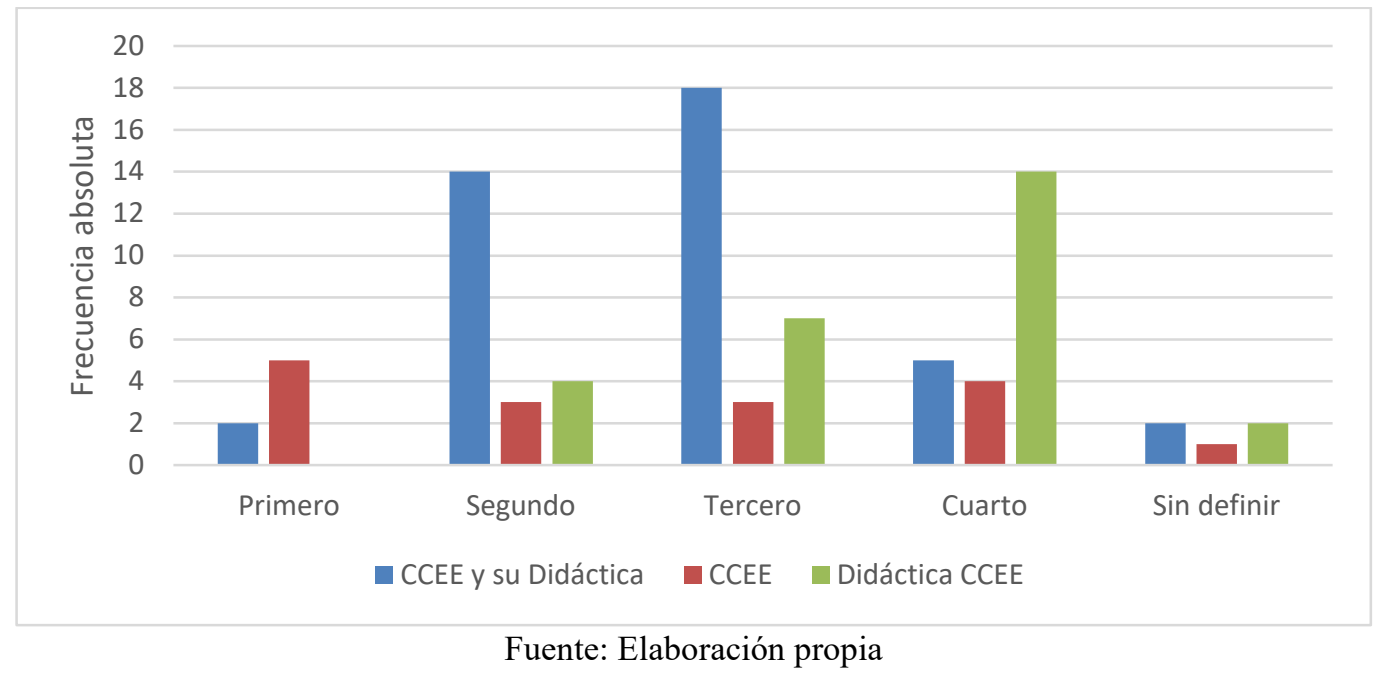

Atendiendo a la variable créditos de las materias podemos decir que 57 asignaturas $(67,9 \%)$ de las analizadas son asignaturas de 6 ECTS y el promedio de créditos obligatorios sobre ciencias experimentales, incluyendo su didáctica, que recibe un estudiante de Grado de Maestro en Educación Primaria es aproximadamente $(16 \pm 4)$ ECTS. Teniendo en cuenta que el total de créditos del grado de Maestro en Educación Primaria es de 240 ECTS, este promedio supone solamente el 6,8\% del total de la formación recibida, dato destacablemente bajo.

En cuanto a la perspectiva, cada universidad oferta un número diferente de asignaturas optativas, de forma que teniendo en cuenta únicamente las asignaturas no optativas de la muestra $(\mathrm{n}=57)$ la mayoría de esta formación obligatoria se recibe en $3^{\circ}$ curso $(36,8 \%)$ seguida de $2^{\circ}$ curso $(35,1 \%)$. La mayoría $(56,1 \%)$ de las asignaturas obligatorias de ciencias son asignaturas que combinan contenidos y didáctica (i.e. CCEE y su Didáctica) y la perspectiva más frecuente es la Genérica (91,2\%), es decir, el contenido impartido hace referencia a varias ramas de la ciencia y no a una específica, ya sea la asignatura de contenido científico o de contenido didáctico. Resultado en línea con el hecho de que se están analizando asignaturas del Grado de Maestro en Educación Primaria, alumnos que después impartirán Ciencias de la Naturaleza. Este último resultado también es similar si tenemos en cuenta todas las asignaturas, tanto las de carácter obligatorio y básico como las optativas, siendo el 92,9\% (78 asignaturas de 84) la presencia de asignaturas impartidas con una perspectiva genérica (tabla 1).

TABLA 1. Distribución de las asignaturas según contenido impartido y perspectiva

\begin{tabular}{rccc}
\hline & CCEE y su Didáctica & CCEE & $\begin{array}{c}\text { Didáctica de las } \\
\text { CCEE }\end{array}$ \\
\hline $\begin{array}{r}\text { Genérica } \\
(\boldsymbol{n}=\mathbf{7 8})\end{array}$ & $38(92,7 \%)$ & $13(81,3 \%)$ & $27(100 \%)$ \\
\hline $\begin{array}{r}\text { Especifica } \\
(\boldsymbol{n}=\mathbf{6})\end{array}$ & $3(7,3 \%)$ & $3(18,8 \%)$ & $0(0 \%)$ \\
\hline Total & $\mathbf{4 1 ( 1 0 0 \% )}$ & $\mathbf{1 6}(\mathbf{1 0 0 \% )}$ & $\mathbf{2 7}(\mathbf{1 0 0 \% )}$
\end{tabular}


Este resultado evidencia la declaración del perfil docente del Maestro en Educación Primaria que propone el libro blanco de la ANECA correspondiente "un perfil de docente generalista, con competencias especificas en las áreas del currículo de Ed. Artística (Exp. Plástica), Matemáticas, Lengua, y Ciencias, Geografía e Historia" (ANECA, 2005, p. 80). Además, tengamos en cuenta que en Primaria no existe la diferencia explícita en las áreas de Física/Química y Biología/Geología, como sí ocurre en cursos superiores. Por ello, entendemos que los resultados obtenidos, en los que la visión genérica de las asignaturas es mayoritaria, van en la línea de defender la integración de todas las ramas de la ciencia para la etapa de primaria, ya sea desde el punto de vista disciplinar como didáctico.

Dentro del cómputo total anterior, se encuentran las asignaturas de la categoría Transversales, que abarca asignaturas como Educación para la Salud, Educación Ambiental, Sostenibilidad y TICs. Este grupo incluye 15 asignaturas y la perspectiva de todas ellas es genérica, dado que son temas transversales y, por lo tanto, necesitan de varias ramas de la ciencia para abordarlas. En cuanto a la distribución según el contenido, 4 de ellas son de Contenidos disciplinares, 5 son de Didáctica y 6 combinan ambas cosas. Asimismo, de las 15 asignaturas de este grupo Transversales, el 73,3\% (11) son asignaturas de carácter optativo, resultado que podría suponer que una gran parte del alumnado finalice el grado sin haber visto estos temas de interés, actuales o globales.

Atendiendo a los datos generales que ofrecen las universidades, sólo 1 universidad oferta únicamente asignaturas de Didáctica de las CCEE (4,8\%), es decir, todas las asignaturas científicas ofertadas por dicha universidad son de Didáctica de las ciencias. No encontramos universidades con únicamente asignaturas de contenidos disciplinares. Este valor obtenido discrepa del encontrado en el trabajo de García Barros (2016), en el que el porcentaje de universidades con enseñanza sólo disciplinar o solo didáctico es del 11,42\% (4 universidades, dos en cada uno de los casos). Entendemos que se debe a las diferencias en la muestra.

\subsection{Segundo objetivo: aparición de palabras clave relacionadas con la indagación en las guías docentes}

Se han analizado 82 guías docentes (las no consideradas fue por no tener acceso a la guía docente o por no encontrarse la guía escrita en castellano o catalán). Los términos de búsqueda que más aparecen son la raíz Experim* (46,3\%) seguida de las raíces Investig* $(36,6 \%)$ y Observa* $(30,5 \%)$. Las palabras que con menos frecuencia encontramos son los ítems Variable y Prueba (con un $2,4 \%$ cada una) y Diseño Experimental, que no aparece en ninguna de las guías estudiadas. Debe comentarse que no se han incluido las palabras que se encontraban en la bibliografía, debido a que hubiera requerido un análisis completo del libro y no sólo del título del mismo. Asimismo, hemos de remarcar la polisemia de algunas palabras anteriores, como investigación, problemas, comunicación o reflexión. Todas las palabras se han considerado únicamente en su contexto, es decir, si hacían referencia como fase de un proceso indagatorio, de forma que aquellas en las que no se explicitaba nada, no se han considerado. Esto ha llevado a la necesidad de, no sólo buscar la palabra clave, sino a realizar un análisis del contexto.

Asimismo, las palabras también aparecen a menudo agrupadas, como por ejemplo en forma de listado de Competencias:

- Observar aspectos estáticos y dinámicos de los fenómenos físico-químicos - Formular hipótesis sobre procesos físicos y químicos. - Medir utilizando instrumentos sencillos. - Identificar y controlar variables. - Clasificar materiales (G027);

o en una misma frase, ya sea en Objetivos de la asignatura:

Adquirir habilidades propias del trabajo cientifico: observar, comparar y clasificar, formular preguntas, formular hipótesis, experimentar, buscar datos y su significado, interpretar los datos y verificarlos, extraer conclusiones y comunicar (G065); 
o dentro del Temario de la asignatura:

Destrezas Procedimentales y su desarrollo en los niños: observación, descripción, formulación de preguntas, formulación de conjeturas e hipótesis, predicciones, explicaciones, razonamiento, medidas y registros, etc. (G075).

Haciendo un promedio del número total de palabras clave que aparecen en las guías docentes estudiadas, encontramos que se incluyen 2,3 palabras por guía, valor destacablemente bajo, teniendo en cuenta que se han buscado 14 términos. Sólo en tres guías $(3,6 \%)$ encontramos al menos la mitad de los términos que hemos estudiado (apareciendo en una de ella 9 y en dos de ellas 8 palabras).

Al realizar el análisis del contenido, se ha tenido en cuenta si las palabras clave hacen referencia sólo al contenido científico o también a una parte didáctica. Para entender esta diferenciación, considérese los siguientes ejemplos. Expresiones como Observación o Habilidades de investigación no parecen aludir a cómo los alumnos del Grado de Maestro aprenden a enseñar la metodología científica, sino más bien, a que ellos asimilen los procedimientos científicos. Este tipo de casos se ha clasificado como únicamente relacionado con el contenido científico. Sin embargo, expresiones como Diseñar investigaciones escolares en el ámbito de los fenómenos físico-químicos o Saber diseñar secuencias de enseñanza basadas en la indagación permiten vislumbrar una parte didáctica, es decir, puede aceptarse que, en estos casos, el alumnado del Grado de Maestro aprende también cómo enseñar y/o explicar los aspectos de la metodología científica a sus futuros alumnos. Estos casos son considerados como que también contienen una parte didáctica. Aun así, debemos destacar que algunas veces, la redacción es demasiado ambigua o escueta.

Así pues, se ha obtenido que la mayoría de palabras encontradas hace referencia solamente al contenido científico, a excepción de la raíz indaga* donde es considerablemente mayor la referencia a Didáctica. Los resultados se muestran en el Gráfico 2, donde destaca también el hecho de que los ítems Datos, Gráfic* y Prueba sólo aparezcan haciendo referencia al Contenido disciplinar y nunca a su parte Didáctica.

GRÁFICO 2. Aparición de las palabras clave clasificadas según Contenido o Didáctica

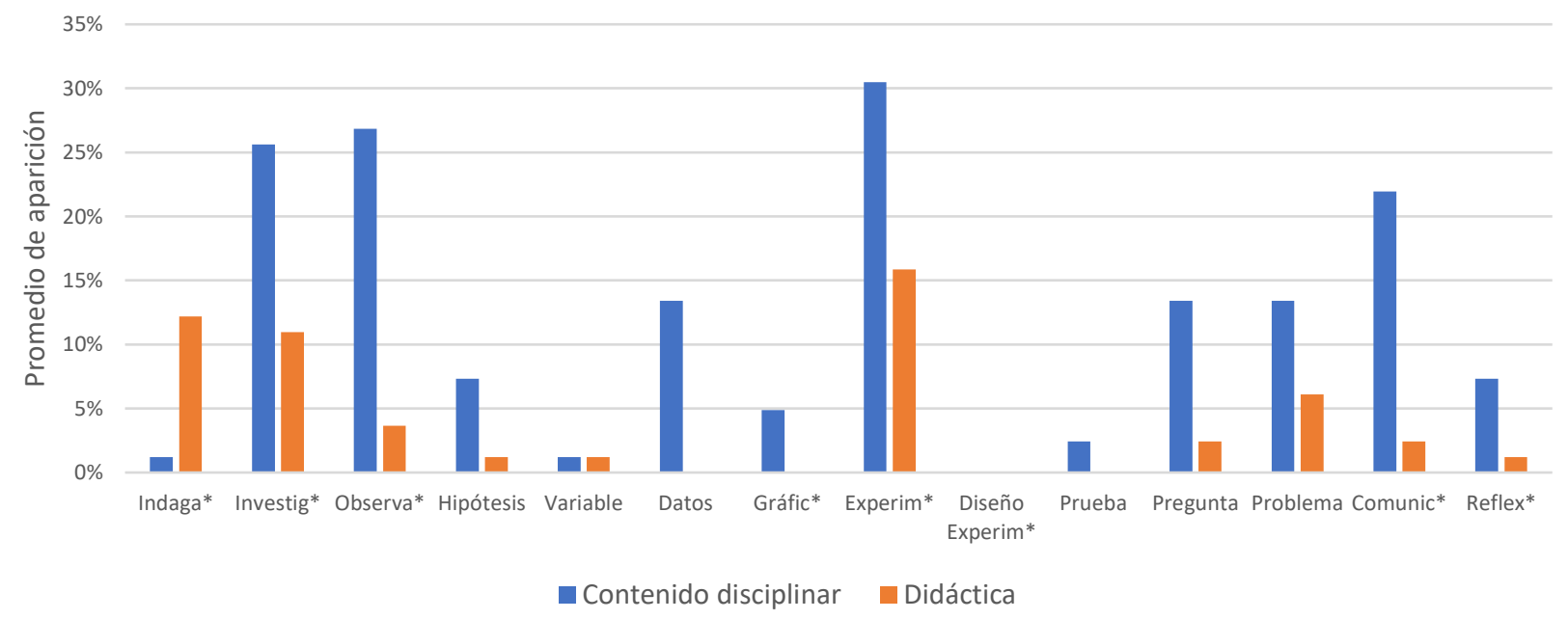

Fuente: Elaboración propia

Del Gráfico 2 se extrae que aparecen más referencias al contenido disciplinar que a la didáctica de las fases del método científico, por lo tanto, estos datos parecen indicar que los futuros maestros reciben formación sobre los procedimientos científicos, pero no sobre la didáctica de los mismos, aunque es cierto que el promedio de 2,3 palabras por guía encontrado nos indica que esta formación es reducida. El resultado acerca de la raíz Indaga* nos plantea una cuestión interesante: aunque la frecuencia de esta palabra no es nula, es posible que no se traduzca en la formación específica de lo 
que significa la indagación, esto es realizar observaciones, plantear hipótesis, identificar variables o diseñar experimentos, dado que el número de apariciones de estos términos es escaso, por lo que presumiblemente la indagación se encuentra más como una declaración de intenciones que como un programa específico a desarrollar.

Por último, se ha estudiado el lugar de la guía docente en el que aparecen las palabras anteriores, atendiendo a si estos ítems hacen referencia al Contenido científico o a su Didáctica. Los lugares de las guías docentes analizadas han sido: Competencias, Resultados de aprendizaje, Temario, Metodología, Evaluación... No se ha incluido la bibliografía en el análisis, de forma que este apartado no se ha considerado en el cómputo final. Incluimos aquí algunos ejemplos de los ítems, el contexto en el que se encuentra y la parte de la guía docente en la que aparece:

- Raíz indag*: Aprendizaje guiado basado principalmente en indagación a través de actividades, intercambio de experiencias y reflexión sobre la práctica (G081), dentro del apartado de Métodos docentes y haciendo referencia a la parte didáctica o ¿Qué es y cómo promover la indagación, la resolución de problemas, la modelización y la comunicación y / o argumentación en el alumnado? (G014) dentro del apartado Temario y considerando también la parte didáctica.

- Raíz observ*: Comprender que la observación sistemática es un instrumento básico para poder reflexionar sobre la práctica y la realidad, así como contribuir a la innovación y a la mejora en educación (G077), dentro del apartado de Competencias y refiriéndose al contenido científico.

- Raíz experim*: Elaborar materiales adecuados para la exploración física del medio natural y tecnológico por los escolares, como por ejemplo montajes experimentales sencillos con finalidades concretas (G075), dentro del apartado de Resultados de aprendizaje y abordando la parte didáctica.

- Raíz hipot*: Conseguir destrezas en el laboratorio: de observación, de planteamiento de hipótesis, de análisis de información, de manipulación del material, etc., que lo hagan competente en el manejo de un laboratorio escolar (G061), dentro del apartado de Objetivos y competencias (Objetivos docentes específicos) y refiriéndose al contenido disciplinar.

- Raíz investig*: Dominio de los conceptos básicos de la disciplina, capacidad de análisis y comprensión, así como la utilización de las técnicas básicas de investigación en ciencias experimentales; también se valorarán las actitudes de trabajo, la iniciativa, colaboración, la implicación y la participación en todas las prácticas de laboratorio y de aula (G055), dentro del apartado de Evaluación del aprendizaje de los estudiantes y abordando únicamente el contenido, no la parte didáctica del mismo.

- Ítem pregunta: Consistirá en la realización de actividades de investigación sobre cada tema; de forma que den respuesta a una pregunta de indagación mediante una toma de datos (G008), dentro del apartado de Evaluación y haciendo referencia también sólo al contenido científico.

- Raíz comunic*: Herramientas digitales para comunicar cientificamente: la pizarra digital interactiva y el lenguaje Scratch (G013), dentro del apartado de Temario y haciendo alusión sólo al contenido científico.

Para el caso de las palabras clave que hacen referencia sólo al contenido científico, es casi anecdótica la presencia de estos términos en el apartado de Temario de la asignatura, a excepción de los ítems Gráfic*, Experim*, Pregunta y Comunic*, cuya aparición en este apartado es más elevada que para el resto de palabras clave, como se puede observar en la Tabla 2. Este resultado difiere de lo que se observa para los ítems que también tratan la Didáctica, cuya aparición en el apartado de Temario es más frecuente en las guías estudiadas, en comparación con la aparición en apartados diferentes. Es interesante el caso de la palabra clave Variable, dado que aparece una vez haciendo referencia al contenido disciplinar y otra a la didáctica y, en ambos casos, el ítem no aparece en el apartado de Temario, por ello, sus porcentajes en la Tabla 2 son idénticos en las columnas. Otros casos similares son el de Investig*, el de Hipótesis y el de Experim*, que también aparecen el mismo 
número de veces en el apartado de Temario aludiendo al contenido disciplinar y a la didáctica $(4,1 \mathrm{y}$ 10 veces, respectivamente).

El que no aparezcan las palabras clave en el Temario pero sí lo hagan en otros apartados puede indicar que, aunque esté la intención de que aprendan los procedimientos de la metodología indagatoria (incluyéndose en apartados como Competencias, Metodología Docente o Resultados de Aprendizaje), realmente no se acabe impartiendo (por no verse reflejado en el Temario). Somos conscientes de la limitación de estos resultados, que deben tomarse sólo como orientativos, puesto que la presencia de estos indicadores no garantiza que se esté impartiendo indagación, pero sí nos da una primera aproximación, pues si no aparecen no se propicia la situación para que ello ocurra.

Es destacable, asimismo, que en lo que respecta al contenido científico, haya mucha más frecuencia de aparición en apartados diferentes al Temario que en dicho apartado (esto ocurre en 12 de las 14 palabras clave analizadas). Esto puede hacer sospechar que los futuros docentes no están recibiendo formación sobre los procedimientos científicos, aunque en su guía docente aparezcan referenciados algunos de ellos. Por el contrario, en lo que respecta a la parte didáctica de las destrezas relacionadas con las etapas de una investigación, 5 de ellas (indaga*, hipótesis, experim*, pregunta y problema) aparecen más frecuentemente en el Temario que en otros apartados, frente a 4 de ellas (investig*, observa*, variable y réflex*) con las que ocurre lo contrario.

TABLA 2 Número de guías (en \%) en las que aparece cada ítem según si hace referencia al contenido científico o a la didáctica, distribuidas a su vez en el lugar de aparición de la guía docente: si lo hace en el apartado de Temario o en otros distintos. Se han marcado en negrita el lugar de la guía que predomina para cada caso.

Referencia al contenido científico

Referencia a la didáctica

\begin{tabular}{|c|c|c|c|c|}
\hline Palabra clave & $\begin{array}{l}\text { Aparece en } \\
\text { Temario }\end{array}$ & $\begin{array}{l}\text { Aparece en otros } \\
\text { apartados }\end{array}$ & $\begin{array}{l}\text { Aparece en } \\
\text { Temario }\end{array}$ & $\begin{array}{l}\text { Aparece en otros } \\
\text { apartados }\end{array}$ \\
\hline Indaga* & $0,00 \%$ & $1,22 \%$ & $7,32 \%$ & $4,88 \%$ \\
\hline Investig* & $4,88 \%$ & $20,73 \%$ & $4,88 \%$ & $6,10 \%$ \\
\hline Observa* & $3,66 \%$ & $23,17 \%$ & $1,22 \%$ & $2,44 \%$ \\
\hline Hipótesis & $1,22 \%$ & $6,10 \%$ & $1,22 \%$ & $0,00 \%$ \\
\hline Variable & $0,00 \%$ & $1,22 \%$ & $0,00 \%$ & $1,22 \%$ \\
\hline Datos & $2,44 \%$ & $10,98 \%$ & $0,00 \%$ & $0,00 \%$ \\
\hline Gráfic * & $3,66 \%$ & $1,22 \%$ & $0,00 \%$ & $0,00 \%$ \\
\hline Experim* & $12,20 \%$ & $18,29 \%$ & $12,20 \%$ & $3,66 \%$ \\
\hline $\begin{array}{r}\text { Diseño } \\
\text { Experim * }\end{array}$ & $0,00 \%$ & $0,00 \%$ & $0,00 \%$ & $0,00 \%$ \\
\hline Prueba & $0,00 \%$ & $2,44 \%$ & $0,00 \%$ & $0,00 \%$ \\
\hline Pregunta & $6,10 \%$ & $7,32 \%$ & $2,44 \%$ & $0,00 \%$ \\
\hline Problema & $3,66 \%$ & $9,76 \%$ & $6,10 \%$ & $0,00 \%$ \\
\hline Comunic* & $3,66 \%$ & $18,29 \%$ & $1,22 \%$ & $1,22 \%$ \\
\hline Reflex* & $0,00 \%$ & $7,32 \%$ & $0,00 \%$ & $1,22 \%$ \\
\hline
\end{tabular}

Fuente: Elaboración propia 


\section{Conclusiones}

Se lleva a cabo un estudio descriptivo y transversal realizado a partir de la información obtenida. La educación científica que reciben los maestros y maestras de Educación Primaria españoles en su formación inicial es analizada en el presente estudio mediante el análisis de la información contenida en las guías docentes de diferentes facultades en las que se imparte dicho Grado

Se ha encontrado que se imparten asignaturas de ciencias experimentales, didáctica de las ciencias experimentales o ambas disciplinas a la vez, predominando el enfoque genérico de todas ellas, es decir, abordando varias ramas de las ciencias experimentales en una misma asignatura, ya sea de contenidos científicos y/o didácticos. Es destacable que la proporción de créditos obligatorios de ciencias experimentales y/o su didáctica recibida por los futuros maestros es muy baja, siendo tan sólo del 6,8\%, lo que no permite recuperar los déficits de su formación previa, teniendo en cuenta que sólo un bajo \% de los mismos ha cursado la modalidad de bachillerato de ciencias y tecnología (un 15,5\% en la UV). La formación en asignaturas de aspectos transversales como TICs o Sostenibilidad se imparte predominantemente en asignaturas optativas, por lo que no todo el alumnado recibe dicha formación.

Por último, la presencia de palabras relacionadas con la indagación en las guías docentes se encuentra de manera desigual, de forma que la raíz experim* y la palabra diseño experimental son los ítems con mayor y menor frecuencia de aparición, respectivamente. Los datos obtenidos parecen indicar que los futuros maestros reciben formación sobre los procedimientos científicos, pero no sobre la parte didáctica de los mismos, ya que la presencia de los ítems estudiados hace referencia mayoritariamente a aspectos únicamente de procedimientos científicos, sin abordar el cariz didáctico. Es destacable, asimismo, que no se encuentre referencia alguna al aspecto didáctico de términos como Datos, Prueba o Gráfic*, aun cuando sí aparecen en lo relacionado al procedimiento como tal. En cuanto al lugar de aparición en las guías, la distribución difiere según se refiera al contenido científico o a la didáctica. En el primero de los casos, los ítems no se encuentran principalmente en el apartado de Temario de la asignatura, a diferencia de los ítems que se refieren a la didáctica, que sí suelen aparecer en dicho apartado. No obstante, la presencia de esta parte didáctica es mucho más baja en las guías estudiadas que la de los ítems que hacen referencia al procedimiento científico, llegando, por tanto, a la contradicción de que la formación de nuestros maestros no incluye apenas cómo enseñar y aprender procedimientos científicos, sino sólo, aunque de forma escasa, conocer y practicar estos procedimientos.

La limitación principal del presente trabajo es la utilización de un único instrumento: la guía docente. En consecuencia, debemos tomar los resultados obtenidos como tentativos, aunque no podemos obviar la puerta que abre la presente investigación: la indagación no parece estar presente en las aulas del Grado en Maestro en Educación Primaria. Somos conscientes de la necesidad de un estudio mucho más amplio que complete el presente para poder analizar el grado real en el que la indagación se imparte hoy en día; un estudio que incluyera entrevistas a alumnos y profesores, observaciones de las lecciones, acceso a las evaluaciones, etc.

Sin embargo, este manuscrito ha analizado la declaración de intenciones de los planes de estudio de los futuros maestros y maestras, el compromiso que establecen los centros universitarios con su alumnado, y la indagación no parece estar presente en ellos de forma evidente. Futuras líneas de investigación pueden nacer de este trabajo, para dar respuesta no sólo a cómo imparten las asignaturas de ciencias los formadores de maestros, sino también qué formación inicial tienen y qué investigaciones en didáctica realizan, ya que estudios recientes en dos países han mostrado su gran influencia en la práctica docente (Solbes y González, 2016; Solbes et al., 2018). Conocer qué imparten, cómo lo hacen y por qué (relacionado con su formación inicial y su experiencia investigadora) puede darnos claves para realizar propuestas efectivas de formación de maestros en ciencias. 
Así, entendemos que la formación científica de los futuros maestros y maestras debe huir de los métodos transmisivos para acercarse a las metodologías en las que el alumnado sea actor principal y responsable de su propio aprendizaje. De la misma manera y, aunque en este trabajo, mayoritariamente hemos encontrado asignaturas que combinan el contenido científico con el didáctico, todavía casi el $20 \%$ de las asignaturas son únicamente de contenidos científicos. Como formadores de maestros y maestras de ciencias, debemos procurar que las asignaturas sean capaces de mostrar la contextualización escolar de la ciencia, así como la integración con la parte didáctica (que muchas veces se impartirá en cursos separados), incluyendo, por supuesto, la indagación. Esta pretensión debemos llevarla a cabo no sólo para una mejor comprensión de los contenidos científicos por parte de nuestro alumnado, sino como una estrategia de enseñanza que podrán utilizar en un futuro para enseñar ciencia. Para ello, trabajos que realmente analicen y lleven la indagación al aula universitaria son necesarios. Propuestas como (Martínez-Chico et al, 2014b; Martínez-Chico, Jiménez Liso y López-Gay, 2015; Vílchez González y Bravo Torija, 2015; Toma et al, 2017) ya marcan el camino a seguir, persiguiendo el propósito de formar docentes en la metodología indagatoria.

En conclusión, la presente radiografía de las asignaturas relacionadas con las ciencias experimentales muestra una formación científica y de didáctica de las ciencias del futuro profesorado de Educación Primaria necesariamente ampliable y con poca presencia de la indagación, de forma que debe abrir un camino de oportunidades para hacer nuevas propuestas de formación que complemente este déficit.

\section{Referencias}

Aguilera Morales, D., Martín-Páez, T., Valdivia-Rodríguez, V., Ruiz-Delgado, Á, Williams-Pinto, L., Vílchez-González, J. M. y Perales-Palacios, F. J. (2018). La enseñanza de las ciencias basada en indagación. Una revisión sistemática de la producción española. Revista de Educación, 381, 259-284. DOI: 10.4438/1988-592X-RE-2017-381-388

Akerson, V. L. y Hanuscin, D. L. (2007). Teaching nature of science through inquiry: Results of a 3year professional development program. Journal of Research in Science Teaching, 44, 653680. DOI: $10.1002 /$ tea.20159

Allen, M. (2010). Misconceptions In Primary Science. Open University Press, Berkshire (Second). ANECA. (2005). Libro Blanco. Título de Grado en Magisterio. Volumen 1. Madrid.

Boletín Oficial del Estado (2014). Real Decreto 126/2014, de 28 de febrero, por el que se establece el currículo básico de la Educación Primaria. BOE núm. 52 de 01/03/2014

Cañal, P. (2000). El conocimiento profesional sobre las ciencias y la alfabetización científica en Primaria. Alambique, 24, 46-56.

Cañal, P. (2012). Saber ciencias no equivale a tener competencia profesional para enseñar ciencias. En E. Pedrinaci (Ed.), 11 ideas clave. el desarrollo de la competencia científica. Barcelona: Graó.

Capps, D. K. y Crawford, B. A. (2013). Inquiry-Based Instruction and Teaching About Nature of Science: Are They Happening? Journal of Science Teacher Education, 24, 497-526. DOI: $10.1007 / \mathrm{s} 10972-012-9314-\mathrm{z}$

Couso, D. (2014). De la moda de "aprender indagando" a la indagación para modelizar: una reflexión crítica. En M. A. Héras, A. Lorca, B. Vázquez, A. Wamba, R. Jiménez. Investigación y transferencia para una educación en ciencias: Un reto emocionante (pp. 1-28). Huelva: Servicio de Publicaciones Universidad de Huelva.

Garrido Espeja, A. y Couso, D. (2017). La modelización en la formación inicial de maestros: ¿qué mecanismos o estrategias la promueven? Enseñanza de las ciencias: revista de investigación y experiencias didácticas, $\mathrm{n}^{\circ}$ extra, 137-144. 
Esteve, J. M. (2006). La profesión docente en Europa: perfil, tendencias y problemática. La formación inicial. Revista de Educación, 340, 19-40.

Forbes, C. T. y Davis, E. A. (2010). Curriculum design for inquiry. Pre-service elementary teachers' mobilization and adaptation of science curriculum materials. Journal of Research in Science Teaching, 47, 820-839. DOI: 10.1002/tea.20379

Furió, C, y Carnicer, J. (2002). El desarrollo profesional del profesor de ciencias mediante tutorías de grupos cooperativos. Estudio de ocho casos. Enseñanza de las Ciencias. Revista de investigación y experiencias didácticas, 20(1), 47-74.

Furtak, E. M., Seidel, T., Iverson, H. y Briggs, D. C. (2012). Experimental and Quasi-Experimental Studies of Inquiry-Based Science Teaching: A Meta-Analysis. Review of Educational Research, 82, 300-329. DOI: 10.3102/0034654312457206

Garbett, D. (2003). Science education in early childhood teacher education: Putting forward a case to enhance student teachers' confidence and competence. Research in Science Education, 33, 467-481. DOI: 10.1023/B:RISE.0000005251.20085.62

Gil, D. (1991). ¿Qué han de saber y «saber hacer» los profesores de ciencias? Enseñanza de las Ciencias. Revista de investigación y experiencias didácticas, 9(1), 69-77.

García Barros, S. (2016). Conocimiento científico conocimiento didáctico. Una tensión permanente en la formación docente. Campo Abierto, 35(1), 31-44. Recuperado de https://mascvuex.unex.es/revistas/index.php/campoabierto/article/view/2825

García Martín, A., (Coord.) (2010). Manual de elaboración de guías docentes adaptadas al EEES: Cartagena. Recuperado de https://repositorio.upct.es/bitstream/handle/10317/1747/isbn9788469350317.pdf;jsessionid=3 204484E68813FFB5CC430ADB463A303? sequence $=1$

Harlen, W. (2009). The Teaching of science in primary schools. (D. Fulton, Ed.) (5th ed., Vol. 5th). Hayes, M. T. (2002). Elementary Preservice Teachers' Struggles to Define Inquiry-based Science Teaching. Journal of Science Teacher Education, 13, 147-165. DOI: 10.1023/A:1015169731478

Keynes, M., Driver, R., Guesne, E. y Tiberghien, A. (1985). Children's ideas in science. Cognition (Vol. 75).

Kirschner, P. A., Sweller, J. y Clark, R. E. (2006). Why minimal guidance during instruction does not work: An analysis of the failure of constructivist, discovery, problem-based, experiential, and inquiry-based teaching. Educational Psychologist, 41, 75-86. DOI: $10.1207 / \mathrm{s} 15326985 \mathrm{ep} 4102 \_1$

Louca, L. T. y Zacharia, Z. C. (2012). Modeling-based learning in science education: Cognitive, metacognitive, social, material and epistemological contributions. Educational Review, 64, 471-492. DOI: 10.1080/00131911.2011.628748

Magnusson, S., Krajcik, L., y Borko, H. (1999). Nature, sources and development of pedagogical content knowledge. En J. Gess-Newsome y N. G. Lederman (Eds.), Examining pedagogical content knowledge (pp. 95-132). Dordrecht, The Netherlands: Kluwer.

Martínez-Chico, M., López-Gay, R., Jiménez Liso, M. R. y Acher, A. (2013). Demandas de maestros en activo y materiales curriculares para la enseñanza de las ciencias. Revista de Investigación en la Escuela, 80, 35-48. DOI: 10.12795/IE.2013.i80.03

Martínez-Chico, M., Jiménez-Liso, M.R. y López-Gay, R. (2014a). La indagación en las propuestas de formación inicial de maestros: análisis de entrevistas a formadores de Didáctica de las Ciencias Experimentales. Enseñanza de las Ciencias, 32(3), 591-608. DOI: 10.5565/rev/ensciencias. 1376

Martínez-Chico, M., López-Gay, R. y Jiménez-Liso, M.R. (2014b). ¿Es posible diseñar un programa formativo para enseñar ciencias por Indagación basada en Modelos en la formación inicial de maestros? Fundamentos, exigencias y aplicación. Didáctica de las ciencias experimentales y sociales, 28, 153-173. DOI: 10.7203/DCES.28.3153 
Martínez-Chico, M., Jiménez-Liso, M.R. y López-Gay, R. (2015). Efecto de un programa formativo para enseñar ciencias por indagación basada en modelos, en las concepciones didácticas de los futuros maestros. Revista Eureka sobre Enseñanza y Divulgación de las Ciencias, 12(1), 149166. DOI: $10498 / 16929$

Mellado, V. (2003). Cambio didáctico del profesorado de ciencias experimentales y filosofía de la ciencia. Enseñanza de las ciencias, 21(3), 343-358. Recuperado de https://www.raco.cat/index.php/Ensenanza/article/view/21943

Minner, D. D., Levy, A. J. y Century, J. (2010). Inquiry-based science instruction-what is it and does it matter? Results from a research synthesis years 1984 to 2002. Journal of Research in Science Teaching, 47, 474-496. DOI: 10.1002/tea.20347

Montero-Pau, J., Tierno, S. P. y Tuzon, P. (2017). Are pre-service Primary School teachers prepared to teach science by inquiry? (pp. 1294-1301). Presentado en 3rd International Conference on Higher Education Advances, HEAd'17, Valencia. DOI: 10.4995/HEAd17.2017.5586

Montero-Pau, J. y Tuzón, P. (2017). Inquiry-based science education in primary school in Spain: teachers' practices. Enseñanza de Las Ciencias: Revista de Investigación y Experiencias Didácticas, extra, 2237-2242. Recuperado de https://0-www-racocat.cataleg.uoc.edu/index.php/Ensenanza/article/view/337605

Murphy, C., Neil, P. y Beggs, J. (2007) Primary science teacher confidence revisited: ten years on. Educational Research, 49(4), 415-430. DOI: 10.1080/00131880701717289

Murphy, C., Smith, G., Varley, J. y Razi, Ö. (2014). Changing Practice: An Evaluation of the Impact of a Nature of Science Inquiry-Based Professional Development Programme on Primary Teachers. Cogent Education, 2(1), 1077692. DOI: 10.1080/2331186X.2015.1077692

Pedaste, M., Mäeots, M., Siiman, L. A., de Jong, T., van Riesen, S. A. N., Kamp, E. T., Tsourlidaki, E. (2015). Phases of inquiry-based learning: Definitions and the inquiry cycle. Educational Research Review, 14, 47-61. DOI: 10.1016/j.edurev.2015.02.003

Porlán, R. (2018). Didáctica de las ciencias con conciencia. Enseñanza de las ciencias, 36(3), 5-22. DOI: $10.5565 / \mathrm{rev} / \mathrm{ensciencias.2795}$

Porlán, R., Rivero, A. y Martín del Pozo, R. (1998). Conocimiento profesional y epistemología de los profesores, II: Estudios empíricos y conclusiones. Enseñanza de las ciencias, 16(2), 271-288. Recuperado de https://www.raco.cat/index.php/Ensenanza/article/view/21534

Rivero, A., Martín del Pozo, R., Solís, E y Porlán, R. (2017). Didáctica de las ciencias experimentales en educación primaria. Madrid: Editorial Síntesis.

Romero-Ariza, M. (2017). El aprendizaje por indagación: ¿existen suficientes evidencias sobre sus beneficios en la enseñanza de las ciencias? Revista Eureka sobre enseñanza y divulgación de las ciencias, 14(2), 286-299. DOI: 10.25267/Rev_Eureka_ensen_divulg_cienc.2017.v14.i2.01

Shulman, L. S. (1986). Those Who Understand: Knowledge Growth in Teaching. Educational Researcher, 15(2), 4-14.

Solbes, J., Domínguez-Sales, M. C., Fernández-Sánchez, J., Furió, C., Cantó, J. R. y Guisasola, J. (2013). ¿El profesorado de física y química incorpora los resultados de la investigación en didáctica? Didáctica de las ciencias experimentales y sociales, 27, 155-178. DOI: 10.7203/DCES.27.2617

Solbes, J., Fernández-Sánchez, J., Domínguez-Sales, M. C. y Cantó, J. R. (2018). Influencia de la formación y la investigación didáctica del profesorado de ciencias sobre su práctica docente. Enseñanza de las Ciencias. Revista de investigación y experiencias didácticas, 36(1), 25-44. DOI: $10.5565 / \mathrm{rev} / \mathrm{ensciencias.2355}$

Solbes, J. y González, E. (2016). Aportes a la formación de profesorado constructivista: resultados en dos países. Praxis \& Saber, 7(13), 63-88. DOI: 10.19053/22160159.4166

Sweller, J., Kirschner, P. y Clark, R. (2007). Why Minimally Guided Teaching Techniques Do Not Work: A Reply to Why Minimally Guided Teaching Techniques Do Not Work: A Reply to Commentaries. Educational Psychologist, 42(2), 115-121. DOI: 10.1080/00461520701263426 
Toma R. B., Greca I. M. y Meneses-Villagrá, J. A. (2017) Dificultades de maestros en formación inicial para diseñar unidades didácticas usando la metodología de indagación. Revista Eureka sobre Enseñanza y Divulgación de las Ciencias, 14(2), 442-457. DOI: 10.25267/Rev Eureka ensen divulg_cienc.2017.v14.i2.11

Verdugo-Perona, J. J., Solaz-Portolés, J. J., y Sanjosé, V. (2016). Pre-service Primary Teachers' Scientific Knowledge and Attitudes towards Science Learning and Their Influence on Understanding of the Nature of Science. Croatian Journal of Education, 18.

Verdugo-Perona, J. J., Solaz-Portolés, J. J., y Sanjosé, V. (2019). Evaluación del Conocimiento Científico en Maestros en formación inicial: el caso de la Comunidad Valenciana. Revista de Educación, 383, 133-162. DOI: 10.4438/1988-592X-RE-2019-383-404

Vílchez González, J. M. y Bravo Torija, B. (2015). Percepción del profesorado de ciencias de educación primaria en formación acerca de las etapas y acciones necesarias para realizar una indagación escolar. Enseñanza de las Ciencias. Revista de investigación y experiencias didácticas, 33(1), 185-202. DOI: 10.5565/rev/ensciencias.1529

Vosniadou, S. (2013). Model based reasoning and the learning of counter-intuitive science concepts. Infancia y Aprendizaje, 36(1), 5-33. DOI: 10.1174/021037013804826519

Windschitl, M. (2003). Inquiry projects in science teacher education: What can investigative experiences reveal about teacher thinking and eventual classroom practice? Science Education, 87(1), 112-143. DOI: 10.1002/sce.10044

\section{CóMO CITAR ESTE ARTÍCULO}

Tierno, S. P., Tuzón, P., Solbes, J. y Gavidia, V. (2020). Situación de la enseñanza de las ciencias por indagación en los planes de estudio de Grado de Maestro de Educación Primaria en España. Didáctica de las ciencias experimentales y sociales, 39, 99-116. DOI: 10.7203/DCES.39.17855 
